\begin{tabular}{c|c|c}
\hline ISSN 2525-4812 (versão online) & Revista Terceira \\
$\begin{array}{c}\text { ISSN 2238-7641 (versão impressa) } \\
\text { http://www.revistaterceiramargem.com/ } \\
\text { index.php/terceiramargem/index }\end{array}$ & $\begin{array}{c}\text { Recebido em: 19/6/2019 } \\
\text { Aprovado em: } 30 / 2 / 2020\end{array}$ & $\begin{array}{c}\text { Revisaço } \\
\text { Margem Amazônia }\end{array}$ \\
\hline
\end{tabular}

Como citar o artigo:

CARVALHO, C. G; FRAXE, T. H. P; SANTIAGO, J. L; CARNEIRO, J. P.R. Segurança alimentar e gestão participativa dos agricultores familiares que atuam no fornecimento de alimentos do PAA Manacapuru (Amazonas, Brasil). Revista Terceira Margem Amazônia, v. 6, n.15, p. 44-60, 2020. Doi: http://dx.doi.org/10.36882/2525-4812.2020v6i15p44-60.

\title{
SEGURANÇA ALIMENTAR E GESTÃO PARTICIPATIVA DOS AGRICULTORES FAMILIARES QUE ATUAM NO FORNECIMENTO DE ALIMENTOS DO PAA MANACAPURU (AMAZONAS, BRASIL)
}

\author{
Claudemar Guarlote de Carvalho ${ }^{1}$ \\ Therezinha de jesus Pinto Fraxe ${ }^{2}$ \\ Jozane Lima Santiago ${ }^{3}$ \\ Janderlin Patrick Rodrigues Carneiro ${ }^{4}$
}

\begin{abstract}
Resumo: Este artigo tem como objetivo verificar se agricultura familiar e as políticas públicas, voltadas à garantia de mercados para a produção familiar, se consolida com a criação do Programa de Aquisição de Alimentos - PAA que tem com um dos seus principais objetivos o fortalecimento da categoria. Nesse contexto verifica-se o programa está promovendo ou não a segurança alimentar nutricional (SAN) e se está contribuindo com a melhoria de renda, qualidade de vida, sustentabilidade econômica dos fornecedores de alimentos da Cooperativa dos Trabalhadores da Agricultura Familiar do Município de Manacapuru - AM (COOTAF). Para isso, na busca pela resposta de qual é o papel que o Programa tem no fortalecimento da agricultura familiar e considerando que o fortalecimento da agricultura também se dá através da organização destes agricultores, em associações ou cooperativas. E nesse sentido, foi feito um estudo em uma organização social, como uma alternativa de mercado, visando apresentar qual é a participação dos agricultores familiares dessa cooperativa com o mercado institucional, a partir disso,
\end{abstract}

\footnotetext{
${ }^{1}$ Administrador de Empresa, Mestre em Ciências do Ambiente e Sustentabilidade na Amazônia pela Universidade Federal do Amazonas - UFAM. Pesquisador do Núcleo de Socioeconomia da Universidade Federal do Amazonas, E-mail: claudemar_guarlott@hotmail.com

${ }^{2}$ Doutora em Sociologia pela Universidade Federal do Ceará (UFC). Professora Titular da Universidade Federal do Amazonas (UFAM). Coordenadora do Núcleo de Socioeconomia. E-mail: tecafraxe@uol.com.br

${ }^{3}$ Doutora em Ciências do Ambiente e Sustentabilidade na Amazônia pela Universidade Federal do Amazonas. Professora Adjunta da Universidade Federal do Amazonas (UFAM). Vice-coordenadora do Núcleo de Socioeconomia. E-mail: jozaneagroecologia@gmail.com

${ }^{4}$ Assistente Social, Mestre em Ciências do Ambiente e Sustentabilidade na Amazônia pela Universidade Federal do Amazonas - UFAM. Pesquisador do Núcleo de Socioeconomia da Universidade Federal do Amazonas, E-mail: patrickcarneiro09@gmail.com
} 
surge o interesse em buscar e coletar dados obtidos através de aplicação de formulários socioeconômicos, questionário sobre a frequência alimentar e aplicada a Escala Brasileira de Insegurança Alimentar - EBIA, que pudessem subsidiar o entendimento sobre a forma com que o PAA contribui para a segurança alimentar dos agricultores familiares.

Palavras-chave: políticas públicas; agricultura familiar; organização social; sustentabilidade

\begin{abstract}
This article aims to verify whether family farming and public policies, aimed at guaranteeing markets for family production, are consolidated with the creation of the Food Acquisition Program - PAA which has as one of its main objectives the strengthening of the category. In this context, it is verified whether the program is promoting nutritional food security (SAN) or not and whether it is contributing to the improvement of income, quality of life, economic sustainability of the food suppliers of the Cooperative of Family Agriculture Workers of the Municipality of Manacapuru - AM (COOTAF). For that, in the search for the answer of what is the role that the Program has in the strengthening of family agriculture and considering that the strengthening of agriculture also occurs through the organization of these farmers, in associations or cooperatives. In this sense, a study was carried out in a social organization, as a market alternative, aiming to present what is the participation of family farmers in this cooperative with the institutional market. From this, there is an interest in seeking and collecting data obtained through application of socioeconomic forms, questionnaire on food frequency and applied the Brazilian Food Insecurity Scale - EBIA, which could support the understanding of the way in which the PAA contributes to the food security of family farmers.
\end{abstract}

Keywords: public policy; family farming; social organization; sustainability

\title{
INTRODUÇÃO
}

Este trabalho irá abordar os resultados da dissertação de mestrado apresentado ao Programa de pós-graduação em Ciências do Ambiente e Sustentabilidade da Universidade Federal do Amazonas, intitulado "Segurança Alimentar e gestão participativa dos agricultores familiares que atuam no fornecimento de alimentos do PAA no Município de Manacapuru - AM", o mesmo mostra as proporções e as formas concretas e os impactos dos programas na qualidade de vida dos Agricultores familiares e sua sustentabilidade.

Desta forma este trabalho mostra como compreender as formas de políticas públicas e sua importância para o desenvolvimento do processo socioeconômico dos produtores e fornecedores do programa de aquisição de alimentos - PAA da cooperativa dos trabalhadores rurais da agricultura familiar - (COOTAF), do município de Manacapuru-AM, e analisar como estão sendo desenvolvidas as políticas públicas e seu 
desempenho no processo socioeconômico e seus reflexos na alimentação adequada e garantia da segurança alimentar e nutricional - SAN, e a sustentabilidade do meio ambiente. Nesse sentido, foi analisado o sistema de gestão participativa dos agricultores fornecedores do programa PAA Doação e observado qual o grau de segurança alimentar após a venda de seus produtos para o PAA Doação, e analisar os impactos na vida desses sujeitos que foram envolvidos no campo da realização do estudo.

Para entender melhor o processo da natureza da pesquisa PAA no Amazonas foi desenvolvido a metodologia, norteada pela pesquisa bibliográfica, com base em referencial teórico, e por meio de visita em campo com aplicação de formulário socioeconômico, quantitativo e qualitativo que teve como objetivo analisar a agregação de valor a partir da organização social, econômica, política e cultural da comunidade, através de implantação de mecanismos sustentáveis e solidário de gestão, comercialização e garantia da qualidade dos produtos e novas formas de trabalho solidário.

O desenvolvimento da pesquisa teve como base uma pesquisa em cima de conhecimento teóricos de autores relevantes ao tema e de diferentes áreas do conhecimento por se tratar de disciplina transdisciplinar e pesquisa documental análise de todos os documentos, elaborados com finalidade diversas (GIL, 2017, p. 30), junto ao Ministério da Agricultura Pecuária e Abastecimento MAPA e CONSEA-AM, visando entender normas e leis especificas e dos processos de certificação participativa, dos produtos agroecológicos; as quais garantem a venda direta dos produtos para o consumidor final, através de feiras e mercados institucionais do programa de aquisição de alimentos (PAA), e identificar as formas de planejamento social qual o planejamento social e solidário aplicado no processo de produção dos produtos agroecológico e a preocupação com o meio ambiente, com o objetivo de fortalecer e garantir credibilidade renda e qualidade de vida

Os resultados foram obtidos por meio de instrumento que foi utilizado para a coleta de dados através de formulário com perguntas abertas e fechadas abordando um levantamento socioeconômico com informações sobre a situação alimentar das famílias de acordo com escala Brasileira (EBIA) e o formulário quantidade de frequência alimentar (QFA), em forma de entrevista, o instrumento é empregado para fins meramente ilustrativo dos registros de campo. As entrevistas foram realizadas nos locais de trabalho com 20 agricultores que fazem parte da cooperativa COOTAF, localizado no Município de Manacapuru-AM. 
ASPECTOS PRODUTIVOS E SOCIOECONOMICOS DOS AGRICULTORES FAMILIARES DA COOPERATIVA DOS TRABALHADORES DA AGRICULTURA FAMILIAR (COOTAF) NO CONTEXTO DO PAA EM MANACAPURU- AM.

Produção da agricultura familiar de acordo com Lamarche (1997), os objetivos a que se propõem os agricultores, para si mesmos e para suas famílias, e conforme também os contextos socioeconômicos locais e o respectivo nível de desenvolvimento, deve-se distinguir as unidades de produção camponesas de outras consideradas de subsistência. Se a função de subsistência está bem presente no modelo camponês, ele não se reduz jamais a isto; há neste modelo, profundamente arraigada, uma vontade de conservação e de crescimento do patrimônio família. (LAMARCHE, 1997).

A produção e a geração das receitas na propriedade da agricultura familiar que foram analisadas, de acordo com gráficos 1, podemos destacar a atividade agrícola desenvolvida pelos agricultores familiares (AF), tivemos um destaque a produção agrícola com ênfase na produção de macaxeira, com (66,100 toneladas), com uma geração de receita de ( $\mathrm{R} \$ 79.320,00)$ seguida do mamão com (20 toneladas), gerando uma receita de $(\mathrm{R} \$ 24,000,00)$ e maracujá com $(8,450$ toneladas) e receita de $(\mathrm{R} \$ 10,140,00)$ acumulando uma receita com esses três produtos um total de $(\mathrm{R} \$ 113,460,00)$ já o faturamento dos demais produtos como: Mandioca, Cebolinha, Coentro, Pimentão, Banana, Pimenta de Cheiro e Cupuaçu geraram uma receita de (R $\$ 20,072,00)$, garantido uma receita liquida para os agricultores no total de $(\mathrm{R} \$ 133.532,00)$ com a comercialização para o mercado institucional do PAA.

Gráfico 1 - Produção dos produtores da agricultura familiar da COOTAF

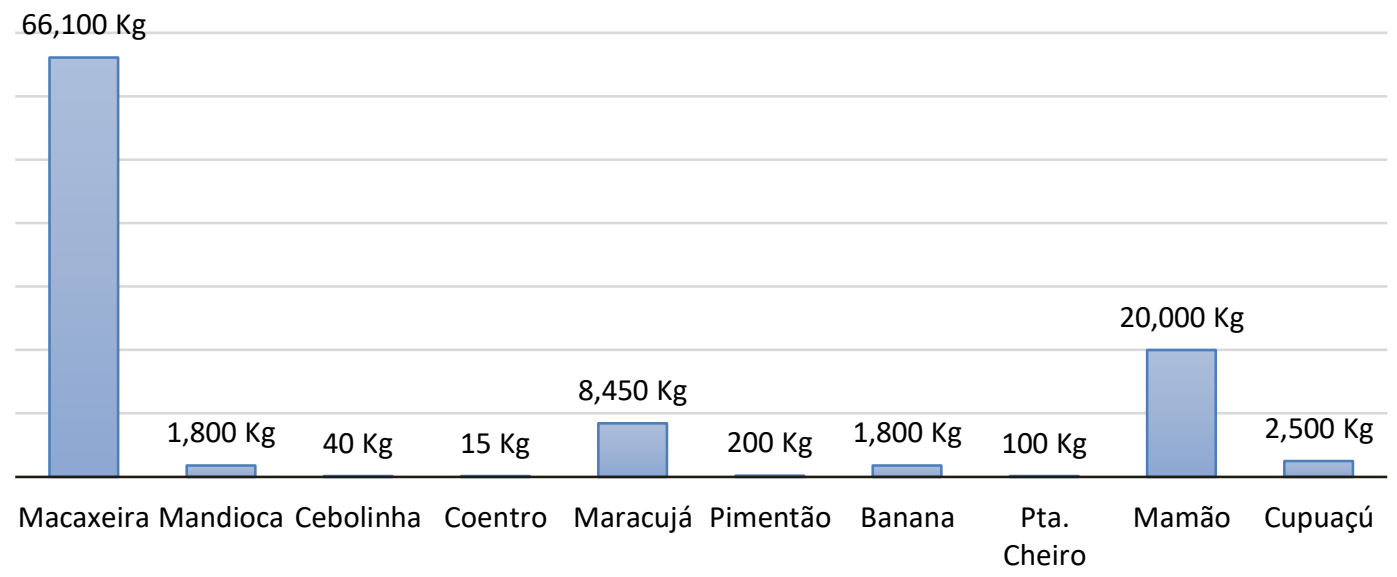

Fonte: Guarlote C.C. (2018) 
No ano de 2018 a COOTAF (Cooperativa dos Trabalhadores da Agricultura Familiar) no município de Manacapuru AM, forneceu para o programa de aquisição de alimentos PAA na modalidade Doação Simultânea, executado peal CONAB - AM 2018, os seguintes produtos:

Tabela 1 - Produtos entregue pela COOTAF para o PAA Doação MDS / CONAB-AM

\begin{tabular}{ccc}
\hline Produtos & Unidade de medida & Peso \\
\hline Produção & Quilograma & Total \\
Banana & $\mathrm{Kg}$ & $2.900 \mathrm{~kg}$ \\
Banana pacovan & $\mathrm{Kg}$ & $2.000 \mathrm{~kg}$ \\
Cupuaçu & $\mathrm{Kg}$ & $1.000 \mathrm{~kg}$ \\
Mamão & $\mathrm{Kg}$ & $13.800 \mathrm{~kg}$ \\
Maracujá & $\mathrm{Kg}$ & $1.900 \mathrm{~kg}$ \\
Melancia & $\mathrm{Kg}$ & $6.313 \mathrm{~kg}$ \\
Pimenta de cheiro & $\mathrm{Kg}$ & $1.500 \mathrm{~kg}$ \\
Pimentão & $\mathrm{Kg}$ & $1.000 \mathrm{~kg}$ \\
Raiz de Macaxeira & $\mathrm{Kg}$ & $38.000 \mathrm{~kg}$ \\
\hline
\end{tabular}

Fonte: CONAB AM (2018).

De acordo com tabela 1 ficou evidente que entre os produtos entregue pelos agricultores da COOTAF, é possível identificar na tabela, os principais produtos produzidos pelos agricultores da cooperativa dentre eles, se destaca a produção de macaxeira. Conforme narrativa de um dos agricultores entrevistados, onde é relatado que todos os cooperados da COOTAF cultivam a macaxeira:

"O nosso principal produto aqui do nosso ramal é a macaxeira todos nos tem roçado de plantio de macaxeira, sem ela eu não consigo viver aqui, quando nós não vende para o PAA o meu filho tem um caminhão ele compra a macaxeira que sobra do contrato da nossa cooperativa e a CONAB, o meu filho compra, ele paga um preço muito bom e ele leva para Manaus, vai vender na Manaus Moderna, e a venda pro meu filho e o PAA ajuda nós aqui no ramal, eu plantava na várzea era ruim quando o rio enchia não dava para arrancar tudo eu perdia muita macaxeira agora aqui na terra firme não tenho esse problema tenho macaxeira o ano todo para vender".

De acordo com a narrativa do agricultor, fica evidente a importância da macaxeira, através da análise dos dados da pesquisa, constatou-se que a macaxeira de fato representa o principal produto dos agricultores familiar dos cooperados da COOTAF, sendo atividade agrícola mais desenvolvida por todos os entrevistados e a principal garantia de renda. 
Segundo PERONI e MARTINS (2000), cultivares contendo altos teores de ácido cianídrico (venenosas) são denominadas mandioca e as com baixos teores (não venenosas) macaxeira, ambas da espécie Manihot esculenta Crantz. A mandioca é um componente básico do sistema de produção agrícola na Amazônia, seja em regiões de terra-firme ou de várzea, devido sua dupla finalidade: autoconsumo e comercialização de farinha de mandioca. É, praticamente, o único produto agrícola que não é comercializado in natura.

\section{PRODUÇÃO DE ALIMENTOS NO MUNCÍPIO DE MANACAPURU-AM.}

O Município de Manacapuru se destaca nas atividades produtivas do Estado do Amazonas se destacando sempre como um dos maiores produtores do Estado, dentre essas atividades produtiva, podemos destacar no cultivo de laranja, cujos os municípios de Rio Preto da Eva, Manacapuru e Manaus são respectivamente os maiores produtores, assim como na produção de Maracujá; Mamão; Mandioca e Banana, ficando entre os maiores produtores de banana do Estado do Amazonas em 2016, se destaca no ranking dos municípios que mais produziram banana que foram: Manacapuru com $(17,75 \%)$, Manicoré (7,93\%), Apuí (7,43\%), Jutaí (4,93\%), Coari (4,18\%), Presidente Figueiredo $(4,32 \%)$ e Rio Preto da Eva (4,47\%). (OLIVEIRA et al, 2017). (IDAM, 2017).

De acordo com Cavalcante (1995) nota-se que essas populações mantêm uma relação de adaptabilidade com o ambiente e seus recursos, desenvolveram conhecimentos, tecnologias, técnicas e processos característicos de um modelo etnoeconômico que fundamenta as suas práticas sociais, culturais e produtivas, particularmente as que dizem respeito às formas de manejo e conservação dos recursos naturais (CAVALCANTI, 1995).

Gráfico 2 - Produção de alimentos para o próprio consumo

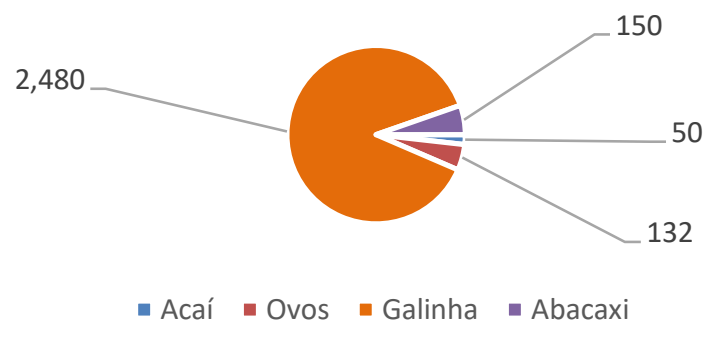

Fonte: Guarlote C.C. (2018). 
A produção dos agricultores analisados dentro das principais atividades agrícolas podemos destacar conforme gráfico 2, os agricultores estão preocupados com sua segurança alimentar e mantem uma produção para o seu próprio consumo, como podemos destacar a produção, açaí 50 litros e 132 ovos que foram produzidos na propriedade dos agricultores e toda essa produção foi para o consumo da sua própria família, dessa forma garantindo uma alimentação saudável e adequada, já a produção de abacaxi com 150 unidades foram comercializados para o PAA no valor de $\mathrm{R} \$ 2,50$ reais a unidade, gerando uma receita com o faturamento de $(\mathrm{R} \$ 375,00)$ com a venda. Porém nesta análise, podemos destacar a produção de Aves ou a produção de galinhas que conforme gráfico 2, a produção foi de 2.480 galinhas sendo que 182 foram para o próprio consumo, isso representa um percentual de $(14 \%)$ da produção usado para o próprio consumo familiar dos agricultores e 2.298 galinhas ou seja ( $86 \%$ ) da produção foram vendidas para o PAA, com o valor de $R \$ 25,00$ reais a unidade viva. Isso é o que equivale uma receita bruta de faturamento de ( $\mathrm{R} \$ 57.450,00)$ com as vendas das galinhas para o programa institucional do governo federal.

Por meio de entrevistas em campo, foi possível observar que os produtores se mostraram muito satisfeitos com a gestão participativa realizadas pela cooperativa através do fornecimento de alimentos para os programas institucionais do governo, onde acarretou uma melhor qualidade de vida para comunidade local, garantindo uma Segurança Alimentar Nutricional -SAN. Através da gestão participativa dos cooperados e a participação afetiva do presidente da cooperativa e seu comprometimento com as políticas públicas, respeitando o meio ambiente e mantendo sempre informados os cooperados com as possíveis mudanças dos programas, e sobretudo a sustentabilidade ambiental.

\section{RENDA FAMILIAR DOS AGRICULTORES DA COOPERATIVA DOS TRABALHADORES DA AGRICULTURA FAMILIAR (COOTAF).}

Para analisar renda dos agricultores foi separada em valores de ( $\mathrm{R} \$ 500,00$ a $\mathrm{R} \$ 3000,00$ ) mensais, isso e que vale de $1 / 2$ salário mínimo a 3 três salários mínimos mensais, isso representa em média por família de produtores analisadas na nossa pesquisa. No âmbito geral do público de 20 produtores analisados, de acordo com gráfico 03 a maior parte dos agricultores possuem uma receita mensal, com a participação das vendas para o PAA, garantindo uma receita mensal de $1 / 2$ a 1 salários mínimos que representa o maior percentual dos entrevistados com (65\%) dos agricultores, seguido do grupo que 
recebe de 1 salaria mínimo e $1 / 2$ salário foi de (15\%), e de 1 e $1 \frac{1}{2}$ salário mínimo mensal se manteve nos (15\%), e como destaque somente um produtor apresentou uma renda mensal de 2 a 3 salário mínimo representando (5\%), dos demais agricultores conforme gráfico abaixo.

Gráfico 3 - Receita dos agricultores familiares fornecedores do Programa de Aquisição de Alimentos COOTAF

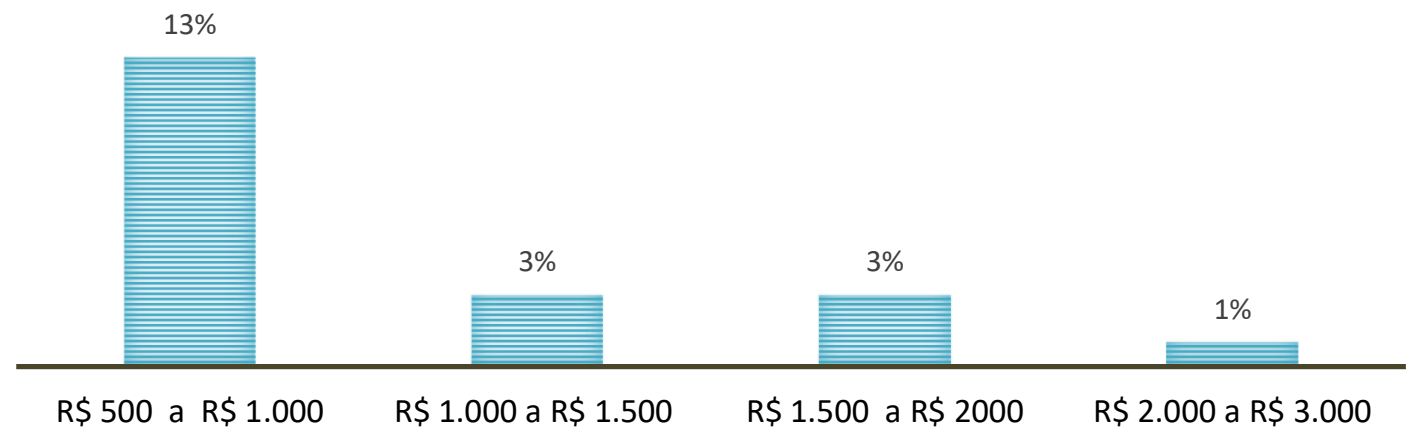

Fonte: Guarlote, C. C. (2018)

Vários estudos indicam que as atividades agropecuárias não são a única fonte de renda existente entre os fornecedores de alimentos ao PAA (MARTINS; CAVALCANTI, 2007; VIEIRA; DEL GROSSI, 2009; HESPANHOL, 2013; GONZAGA, 2015), mas essas atividades são muito importantes para a receita das famílias, porém ela é a principal fonte de renda das famílias dos agricultores familiar.

As políticas públicas do governo federal por meio do PAA, é uma fonte de garantia de renda e fortalecimento da agricultura familiar, que tem como objetivo de garantir uma melhor qualidade de vida aos agricultores e principalmente fomentar o mercado fortalecendo à agricultura familiar. Políticas públicas estruturadas "têm efeitos importantes para a diminuição da vulnerabilidade alimentar das famílias, por meio do aumento da renda familiar, da universalização dos direitos sociais e do acesso à alimentação de qualidade e para a diminuição da desigualdade de renda" (GRAZIANO DA SILVA; DEL GROSSI; FRANÇA, 2010, p. 21).

Nesse contexto os agricultores também foram consultados sobre as principais fontes de renda, de acordo com o gráfico 4 abaixo. A frequência das respostas ficou distribuída conforme o gráfico abaixo, Bolsa família (21\%), Aposentadoria previdência social (26\%), pensão (5\%), ajuda familiar (11\%), não recebe nem um tipo de benefício (37\%), isso só reforça a nossa análise feita em campo, pois os agricultores na sua maioria 
dos entrevistados (37\%) não recebe qualquer tipo de ajuda financeira, ou seja, vivem da receita adquiridas através da produção agrícola que fornecem para o PAA, isso nos leva a uma reflexão sobre a importância do programa para esses agricultores que nesse caso, é de fundamental importância, tendo em vista que é a principal e a maior fonte de renda da sua família, mas de acordo com a pesquisa não representa a única fonte de renda dos agricultores.

Gráfico 4 - Agricultores familiares que recebem algum benefício governamental.

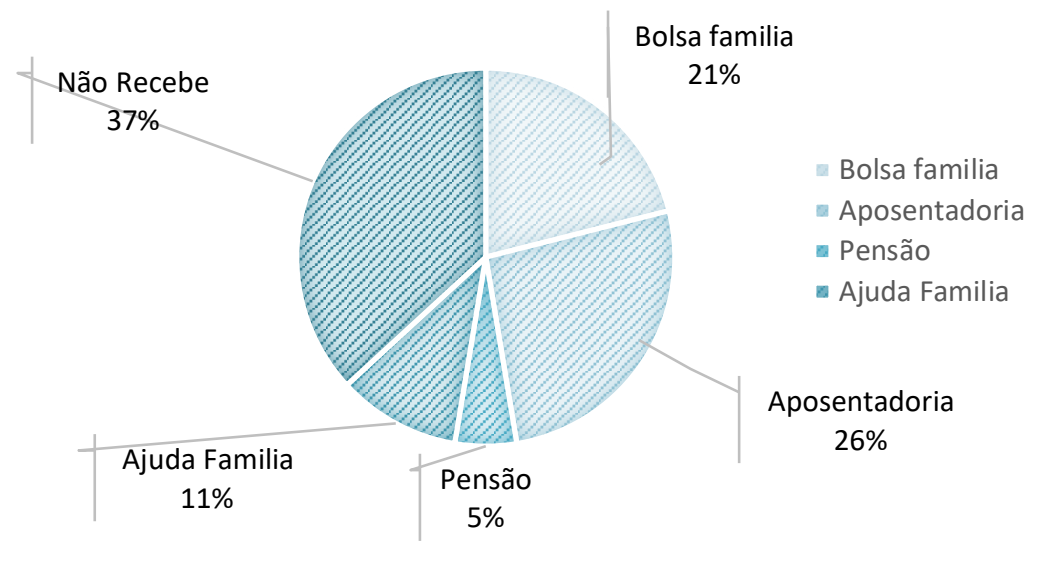

Fonte: Guarlote C. C. dados da pesquisa, (2019).

O PAA possibilita um aporte financeiro considerado pequeno, (10\%) da renda do produtor ao ano, mas é justamente essa pequena contribuição que somada a outras fontes (bolsa família, aposentadoria, trabalho assalariado) complementa e eleva sua renda total (HESPANHOL, 2013).

\section{SEGURANÇA ALIMENTAR E NUTRICIONAL DOS AGRICULTORES FAMILIAR}

O consumo de alimentos adequados e não adequados consumido pelos agricultores, foram avaliados através do Questionário de Frequência Alimentar (QFA) baseado nos marcadores do Sistema de Vigilância Alimentar e Nutricional (SISVAN) para indivíduos com cinco anos de idade ou mais, proposto pelo Ministério da Saúde. Essa ferramenta objetiva identificar a frequência de consumo de alguns alimentos e/ ou bebidas que estão relacionados tanto a uma alimentação saudável como não saudável em determinado período essa ferramenta foi utilizada na pesquisa desenvolvida por Bastos 
(2014) para estimar alimentação adequada da qualidade alimentar de famílias rurais no Distrito Federal.

Conforme dados apresentados no gráfico 5 observamos que existe uma situação de alimentação inadequada a Insegurança Alimentar e Nutricional (ISAN), a mesma também foi analisada em nossa pesquisa. No entanto, foi possível identificar através dos indicadores do consumo de alimento semanal e o consumo diário dos fornecedores de alimentos, tivemos um médio consumo dos alimentos considerados inadequados, de acordo com Bastos (2014), e tudo o que se come e se bebe é ainda, em grande parte, uma questão familiar e social que pode gerar e levar a uma prevalência de ISAN dos agricultores e o consumo, de: (embutidos, biscoitos e/ou doces, bolachas salgadas, salgadinhos de pacote e/ou macarrão instantâneo, refrigerantes, sucos industrializados e bebida alcoólica). (BASTOS 2014.).

Gráfico 5 - Consumo de alimentos adequados e inadequados dos agricultores familiar

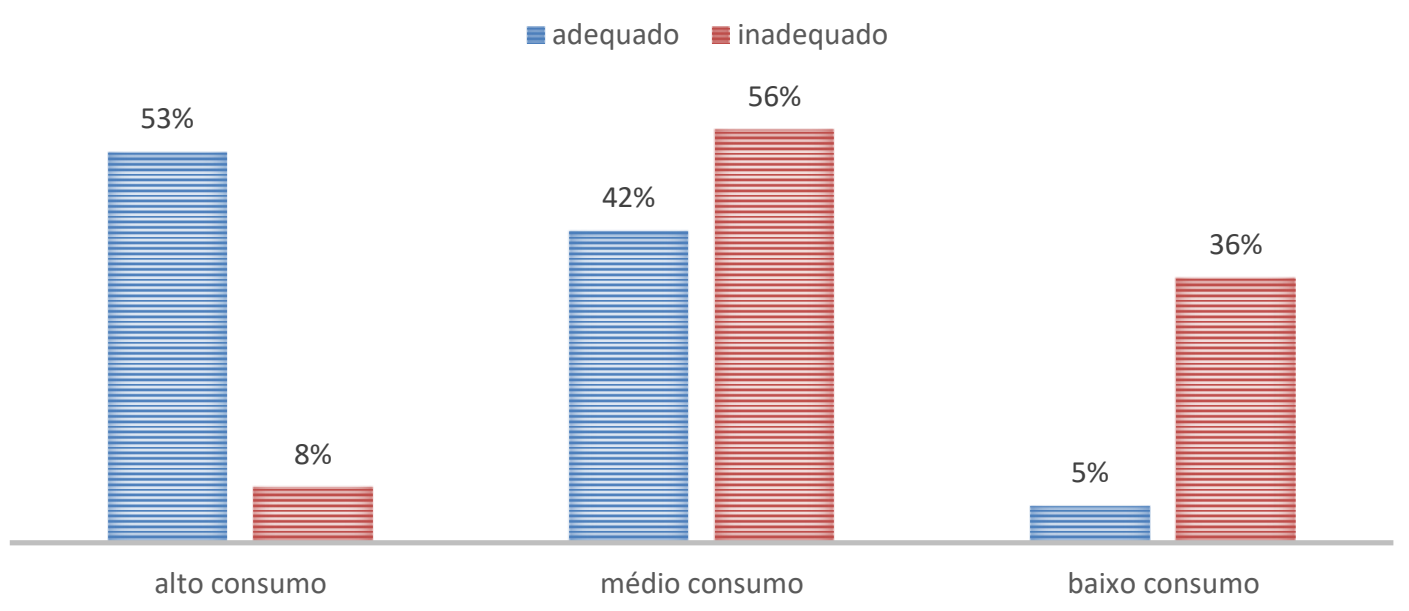

Fonte: Guarlote C.C dados da pesquisa (2019).

Para obtermos uma melhor avaliação utilizamos as categorias de não consome (denominada baixo consumo). Posteriormente, o consumo alimentar foi classificado em consumo adequado para os alimentos consumidos entre 1 a 7 vezes na semana de (alto consumo), alimentos considerados adequados e saudáveis: legumes e verduras, frutas, feijão e/ou leguminosas, leite e/ou derivados, cereais e pães, tubérculos e raízes e carnes, e para o consumo inadequado dos alimentos não saudáveis consumidos entre 1 a 7 vezes na semana, (médio consumo) para os alimentos considerados inadequados que de acordo com Bastos (2014). Em relação aos grupos alimentares saudáveis legumes/verduras e frutas, foi considerado o consumo adequado aquele igual ou superior a cinco dias na 
semana, segundo o recomendado pela literatura brasileira (MOURA; SILVA; MALTA; NETO, 2011).

A frequência do consumo alimentar familiar de acordo com as categorias de baixo, médio e alto consumo, classificou-se o consumo de adequado e inadequado, as variações de consumo de alimentos dos agricultores com uma proporção de garantia de alimentos saudáveis ou adequados com (53\%) dos agricultores que tem um alto consumo de alimentos considerados saudáveis no consumo semanal, desse modo garantindo uma segurança alimentar nutricional (SAN) dos agricultores, porém através do aumento da renda familiar houve um aumento na frequência alimentar de (56\%) dos agricultores que desenvolveram hábitos alimentares não saudáveis ou inadequados que podem comprometer a sua saúde e de sua família e levar a uma insegurança alimentar nutricional de (INSAN).

De acordo com o gráfico 5 pode-se constatar que agricultores entrevistados já não tem hábitos de consumirem com certa frequência os alimentos que produzem, produtos que garante uma alimentação adequada e saudável, ou seja in natura, porém, $(75 \%)$ afirmaram que ingerem refrigerante, seguido de (50\%) dos que consume bebida alcoólica, (40\%) consumem alimentos embutidos, (35\%) dos entrevistados falaram que consumem alimentos bolacha salgadas, salgadinhos de pacote e macarrão instantâneo; (30\%) falaram que consumem biscoitos e doces e (30\%) dos entrevistados consumem sucos industrializados na semana, esse indicador aponta a necessidade de promover políticas de segurança alimentar (SAN) que estimulem hábitos e o consumo alimentar saudáveis e que mantenha os agricultores familiares informados sobre o assunto e sobre alimentação saudável e adequada.

O resultado pode caracterizar uma mudança nos hábitos alimentares dos agricultores da zona rural, porém os agricultores apresentam maior frequências no consumo alimentar de alimentos não saudáveis (embutidos, biscoitos e/ou doces, bolachas salgadas, salgadinhos de pacote e/ou macarrão instantâneo, refrigerantes, sucos industrializados e bebida alcoólica). ADAMS e PIPERATA (2014), observaram que as alterações no consumo alimentar de agricultores familiares da Amazônia brasileira foram ocasionadas pela urbanização, pelo aumento de renda e devido ao acesso e estímulo ao mercado de consumo. 
CARVALHO, C. G; FRAXE, T. H. P; SANTIAGO, J. L; CARNEIRO, J. P.R

Gráfico 6 - Frequência alimentar de SAN e ISAN dos fornecedores da COOTAF

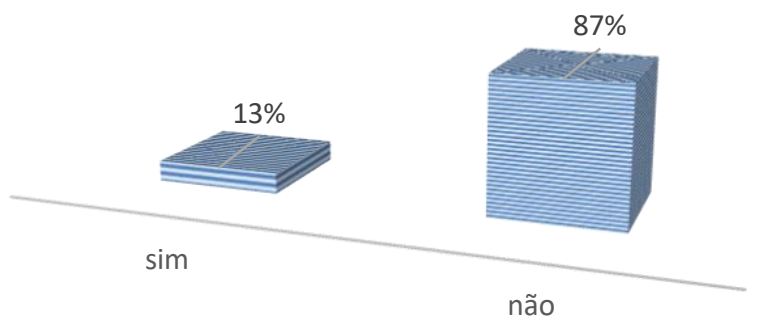

Fonte: Guarlote C.C; dados da pesquisa (2018)

Conforme gráfico 6 observou-se uma prevalência de SAN com mais de (87\%) dos entrevistados responderam não, de acordo com a escala EBIA, é possível a firma que os agricultores familiar da COOTAF fornecedoras de alimentos ao PAA, possuem menor incidência de insegurança alimentar, mas mesmo assim ainda tivemos um percentual de (13\%) dos entrevistados responderam sim, apresentando assim alguma incidência de ISAN, dessa forma o programa PAA tem se tornado uma fonte de renda e garantia de alimentação para os fornecedores desse programa, mas mesmo assim ainda tivemos uma pequena incidência de ISAN, isso demonstra que os agricultores familiares devem estrutura-se em ações públicas no setor da alimentação e nutrição, no contexto da segurança alimentar e nutricional para garantir o direito humano a uma alimentação adequadas dos agricultores. Esse rsultado nos leva a refletir que os programas do governo na sua íntegra, quando é bem gerenciado torna a vida das pessoas mais digna e próspera.

Nesse sentido nos faz concorda com NARDOTO et al. (2011) em pesquisa sobre os hábitos alimentares de ribeirinhos constatou que os mesmos deixaram de comer alimentos tradicionais e passaram a se alimentar com produtos industrializados como a carne bovina e alimentos com altos teores de açúcar alimentos que consumidos com certa frequência podem comprometer a saúde dos agricultores.

No Amazonas, a SAN de acordo com pesquisa do IBGE nos domicílios foi reduzida de $(66,9 \%)$ em 2009 para $(57,1 \%)$ em 2013 uma redução de $(9,8 \%)$ nesse período, isso representa um SAN segurança alimentar positiva pois de acordo com a escala EBIA quanto mais próximo de zero a SAN está sendo mantida. (PNAD/IBGE, 2013).

A ISAN é a falta de disponibilidade e o acesso aos alimentos que tem como critério as perguntas conforme quadro 1 , voltadas para a restrições, no que tange à quantidade e 
acesso aos alimentos dos agricultores familiares da COOTAF, entrevistados na nossa pesquisa, para isso utilizou-se como ferramenta uma proposta da versão curta da EBIA, validada por Santos (2014) onde a situação de ISAN é avaliado conforme as respostas dadas pelos entrevistados. Resposta positiva representa 1 (um) ponto, resposta negativa representa pontuação 0 (zero). O somatório das respostas pode variar de 0 a 5 . Somatório igual a 0 (zero) significa que a família está em situação de SAN. Valores totais acima de 1 significa que a família está em condição de ISAN (SANTOS et al., 2014).

Quadro 1: Escala EBIA versão aplicada aos fornecedores COOTAF - Manacapuru -AM

\begin{tabular}{|c|l|}
\hline & \multicolumn{1}{c|}{ PERGUNTAS } \\
\hline 1 & $\begin{array}{l}\text { Nos últimos } 3 \text { meses o(a) Sr(a) teve a preocupação de que a comida na sua casa acabasse antes que tivesse } \\
\text { condição de comprar, receber ou produzir mais comida? }\end{array}$ \\
\hline 2 & Nos últimos 3 meses a comida acabou antes que o(a) Sr(a) tivesse dinheiro para comprar mais? \\
\hline 3 & Nos últimos 3 meses, o(a) Sr(a) ficou sem dinheiro para ter uma alimentação saudável e variada? \\
\hline 4 & $\begin{array}{l}\text { Nos últimos } 3 \text { meses, o(a) Sr(a) ou algum adulto em sua casa diminuiu, alguma vez, a quantidade de } \\
\text { alimentos nas refeições, ou pulou refeições, porque não havia dinheiro suficiente para comprar a comida? }\end{array}$ \\
\hline 5 & $\begin{array}{l}\text { Nos últimos 3 meses, o(a) Sr(a) alguma vez comeu menos do que achou que devia porque não havia } \\
\text { dinheiro suficiente para comprar comida? }\end{array}$ \\
\hline
\end{tabular}

Fonte: Santos et al., (2014)

A EBIA é um modelo de questionário americano que foi adequado e validado no Brasil para famílias urbanas e rurais. Esse indicador é utilizado pelo IBGE na PNAD desde 2003, demonstrando a importância e a consistência do indicador. A Escala avalia a SAN e a ISAN dos últimos três meses anteriores à pesquisa, examinando a capacidade de acesso das famílias às refeições a alimentos nos aspectos qualitativos e quantitativos, incluindo a percepção da capacidade de garantir o acesso aos alimentos num futuro próximo, assim como a percepção de fome, e associação com os indicadores sociais e nutricionais. (SEGALL-CORRÊA et al., 2003; SEGALL -CORRÊA, 2007; PÉREZESCAMILLA, SEGALL-CORRÊA, 2008; SEGALL-CORRÊA; MARIN-LEON, 2009; KEPPLE; SEGALL-CORRÊA, 2011; AQUINO et al., 2014).

\section{CONSIDERAÇÕES FINAIS}

O resultado da pesquisa demonstrou a importância das políticas públicas para o desenvolvimento do processo socioeconômico dos produtores e fornecedores do PAA na 
cooperativa dos trabalhadores rurais da agricultura familiar - COOTAF do Município de Manacapuru-AM.

No mesmo sentido, os agricultores envolvidos na pesquisados possui conhecimento a respeito da sustentabilidade do meio ambiente, e da importância da sua permanência no meio rural enquanto fornecedores de alimentos para os programas institucionais e sabem da relevância que o PAA proporciona para uma melhor condição de vida dos agricultores familiares.

A pesquisa identificou a importância do PAA para a manutenção das questões sociais e a possibilidade da sucessão familiar, observamos que os agricultores entrevistados, relataram que os seus filhos poderão continuar vivendo na comunidade e ter uma melhor qualidade de vida devido as políticas públicas dos proporcionada pelos mercados institucionais disponíveis para pequenos agricultores, pois segundo narrativa dos agricultores familiares entrevistados o PAA garante uma renda certa por meio do fornecimento de alimentos, com isso é possível incentivar os jovens e evitar o êxodo rural nas comunidades, consolidando o processo produtivo da agricultura familiar.

Esta característica vem ao encontro com o relato de Triches (2012), onde a autora afirma que os conceitos relativos à questão da saúde e ambiental e a busca do estabelecimento de relações de confiança não são mais pautados na formalidade da indústria, mas nas relações de proximidade com os produtores. Estas demandas, por sua vez, são utilizadas pelos produtores para inovar em suas estratégias de reprodução social. (TRICHES, 2012).

Entender a sustentabilidade e as políticas públicas, como uma ferramenta e analisar até que ponto o PAA pode contribuir com o fortalecimento da agricultura familiar, foi o maior desafio enfrentado para a conclusão desse artigo. Portanto afirmar que as políticas públicas desenvolvidas para o fortalecimento da agricultura familiar, quando bem direcionada podemos afirmar que garante uma melhor qualidade de vida dos agricultores, e principalmente fortalece a permanência dos seus familiares na comunidade e com isso garantir o fortalecimento e a sustentabilidade da agricultura familiar.

\section{REFERÊNCIAS}

ADAMS, C; PIPERATA, B. A. Ecologia Humana, Saúde e Nutrição na Amazônia In: Vieira; Toledo; Santos Jr. Ambiente e sociedade na Amazônia: uma abordagem interdisciplinar, 2014. No prelo. 
BASTOS, C. M. M. Insegurança alimentar e nutricional e fatores associados em famílias do núcleo rural agrícola Lamarão, no Distrito Federal. 2014. 110 f. Dissertação (Mestrado em Nutrição Humana) - Universidade de Brasília, Faculdade de Ciências da Saúde, Brasília, 2014.

BRASIL (2009e). MINISTÉRIO DA AGRICULTURA, PECUÁRIA E ABASTECIMENTO. Instrução Normativa $\mathbf{N}^{\mathbf{0}} \mathbf{5 0}$, de 5 de novembro de 2009. Institui o selo único oficial do Sistema Brasileiro de Avaliação da Conformidade Orgânica. Diário oficial da União, Brasília, 06 de novembro de 2009. Seção 1, p. 5

CAVALCANTI, Clovis. Desenvolvimento e Natureza: Estudos para uma Sociedade Sustentável. São Paulo: Cortez, 1995. 429 p.

CAISAN- Câmara Intersetorial de Segurança Alimentar e Nutricional do Amazonas.

Plano Estadual de Segurança Alimentar e Nutricional - 2018 Disponível em: http://www.seas.am.gov.br/wpcontent/uploads/2018/12/Plano_Estadual_de_Seguranca_ Alimentar_e_Nutricuonal_2018-2019_WEB-compressed.pdf. Acessado em 15 Abril de 2019.

CONAB. Companhia Nacional de Abastecimento. Transparência do PAA da CONAB

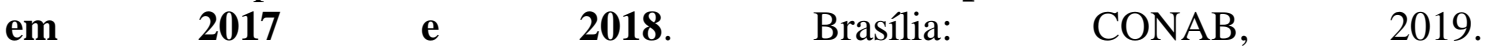
http://Consultaweb.conab.gov.br/consultas/consultatransparenciapaa.do?method acessado em 26 Abril 2019.

GIL, A. C. 1946 - Como Elaborar Projetos de Pesquisa / Antonio Carlos Gil $5^{\text {a }}$ edição São Paulo. Editora Atlas 2017.

GRAZIANO DA SILVA, J.; DEL GROSSI, M. E.; FRANÇA, C. G. Fome Zero: a experiência brasileira. Brasília: MDA, 2010. 360p.

IDAM: Instituto de Desenvolvimento Agropecuário e Florestal Sustentável do Estado do Amazonas. Relatório de Acompanhamento Trimestral, 2017. Disponível em: www.idam.am.gov.br/wp-content/uploads/2018/05/Produção-Florestal-2017.pdf.

Acessado 15 de Abril 2019.

LAMARCHE, Eughes. A agricultura familiar: comparação internacional. Campinas: Unicamp, 1997. 2.ed.

MALTA, D. C. et al. Fatores de risco e proteção para doenças crônicas não transmissíveis entre beneficiários da saúde suplementar: resultados do inquérito telefônico Vigitel, Brasil, 2008. Ciência \& Saúde Coletiva, v. 16, n. 3, p. 2011-2022, 2011

PERONI, Nivaldo; MARTINS, Paulo Sodero. Influência da dinâmica agrícola itinerante na geração de diversidade de etnovariedades cultivadas vegetativamente. Interciência. $v$. 25, n. 1, p. 22-29, 2000.

PNAD: IBGE. Insegurança Alimentar nos Domicílios do Brasil - 2013 https://www.ibge.gov.br/home/estatistica/populacao/seguranca_alimentar_2013/

SANTOS, L. P. S. Proposta de versão curta da Escala Brasileira de Insegurança Alimentar. Revista de Saúde Pública, v. 48, n. 5, p. 783-789, 2014 b. 
HESPANHOL, Rosangela Aparecida de Medeiros. Programa de Aquisição de Alimentos: limites e potencialidades de políticas de segurança alimentar para a agricultura familiar. Sociedade \& Natureza, Uberlândia, v. 3, n. 25, p.469-483, set./dez. 2013.

TRICHES, Rosane M., O Papel da Qualidade dos Alimentos na Construção de Mercados Locais: O Caso da Alimentação Escolar. 50 Congresso da SOBER. Anais. Vitória, 2012.

BRASIL Lei de Segurança Alimentar e Nutricional. Conceitos. Lei 11.346, de 15 de setembro de 2006. Brasília, outubro de 2006.

BRASIL. Lei 11.326, de 24 de Julho de 2006. Estabelece as diretrizes para a formulação da Política Nacional da Agricultura Familiar e Empreendimentos Familiares Rurais. Diário Oficial da União, Brasília, 25/07/2006.

SEGALL-CORRÊA, A. M.; MARIN-LEON, L. A Segurança Alimentar no Brasil: Proposição e Usos da Escala Brasileira de Medida da Insegurança Alimentar (EBIA) de 2003 a 2009. Revista de Segurança Alimentar e Nutricional, Campinas, v.16, n.2, p. $1-19,2009$.

MARTINS, S. P.; CAVALCANTI, L. I. Avaliação do impacto da execução do PAA no Estado do Rio Grande do Norte. Sociedade e desenvolvimento rural, v. 1, n. 1, p. 1-30, 2007

NARDOTO, G. B. et al. Frozen chicken for wild fish: nutritional transition in the Brazilian Amazon region determined by carbon and nitrogen stable isotope ratios in fingernails. American Journal of human biology, v. 23, n. 5, p. 642-650, 2011. 